\title{
Time Dependence of the Output Signal Morphology for Nonlinear Oscillator Neuron Based on Van der Pol Model
}

\author{
Vasyl Lytvyn, Victoria Vysotska, Ivan Peleshchak, Ihor Rishnyak \\ Information Systems and Network Department, Lviv Polytechnic National University, Lviv, Ukraine \\ E-mail: Vasyl.V.Lytvyn@1pnu.ua, Victoria.A.Vysotska@1pnu.ua, peleshchakivan@gmail.com, \\ Ihor.V.Rishnyak@lpnu.ua \\ Roman Peleshchak \\ Ivan Franko Drohobych State Pedagogical University, Department of General physics, Drohobych, Ukraine \\ E-mail: rpeleshchak@ukr.net
}

Received: 05 July 2017; Accepted: 21 October 2017; Published: 08 April 2018

\begin{abstract}
Time-frequency and time dependence of the output signal morphology of nonlinear oscillator neuron based on Van der Pol model using analytical and numerical methods were investigated. Threshold effect neuron, when it is exposed to external non-stationary signals that vary in shape, frequency and amplitude was considered.
\end{abstract}

Index Terms-Nonlinear oscillator neuron, frequency modulation, morphology of the information signal, resonance effect, encoding and decoding of information.

\section{INTRODUCTION}

At present, intensive research on the application of neural networks is being conducted to solve a wide range of Data Mining tasks (identification of non-stationary chaotic processes, clusterization, classification, intellectual management, biosystems states diagnostics, prediction, emulation and recognition of multispectral input images). Considerable interest in the study of modern neurodynamics lies in the processes of information encryption, decryption and processing that is transmitted by neurons. In the early stages of sensory information processing Wavelet analysis is an effective tool to study the information component of the neural signals that are registered. Traditionally such research demands to analyze the structure of point processes, i.e. the time-frequency dynamics analysis of neural reviews [1]-[6], in which information carriers are times of pulses (spikes) generating, but not their form [7]. The mechanisms that lead to the spikes generation are partially known [8]. But how neurons and their ensembles transmit information about around world, so far has practically been unexplored.

In the wavelet-neurophase network, the waveletneuron is structurally close to the standard formal neuron with $N$ inputs, but instead of the usual synaptic weights $\lambda_{i k}$, there are wavelet-synaptic weights $\lambda_{i k}^{W S_{i}}$ $(i=1,2, \ldots, N)$, in which the adjustable parameters are not only the weights $\lambda_{i k}$, but also the scaling and offset wavelets. It should be noted that in all of the abovementioned artificial neural networks, neurons were considered without their own dynamics (their own frequency of oscillations of the neuron $\omega_{0 i}=0$ ) and without taking into account the accumulation of impulses $N_{0 k}$ in the neurons that triggered when $N_{0 k} \geq N_{c k}$ ( $N_{c k}$ is the threshold value of the k-th neuron pulses). The main advantages of self-developing artificial nonlinear neural networks is the ability to adapt to dynamic conditions and the speed of functioning, which is especially important when operating in real-time. In order to provide the highest class of efficiency (speed of operation) and the quality of the recognition of input spectral images, it is proposed to develop the architecture of self-developing artificial nonlinear neural networks and an algorithm for their training based on evolutionary simulation methods that can adapt to dynamic conditions when working in real-time.

Accordingly, to above-mentioned there is necessity to solve the following problems:

- It is necessary to suggest the method of inhomogeneous nonlinear differential equations with quadratic non linearity of the unknown function at the first derivative.

- It is necessary to define that nonlinear oscillator neuron can act as a frequency modulator, which can modulate the input information of nonstationary signal.

- It is necessary to determine that nonlinear oscillator neuron with threshold effect significantly alters the structure of the input information non-stationary signal different in shape, frequency and amplitude. 
- It is necessary to determine the existence of resonance effects in nonlinear oscillator neuron when frequency of the external non-stationary signal and dynamics of the natural frequency of the neuron are equal.

- It is necessary to suggest information coding with nonlinear oscillator neuron on the basis of frequency modulation and decoding using an inverse operator, which acts on the output signal vector.

\section{RELATED WORKS}

In [2], [3], [6], the authors analyzed the case of signals conversion by sensory neurons (threshold device), but its own dynamic neuron was not taken into consideration. Using classical models threshold systems such as "integrate-and-fire" [2] and "threshold crossing" [3], [6], it has been shown that various characteristics of complex dynamics at the entrance sensory neuron are stored in the point process structure [2]-[6], [9]-[11], [17]-[33].

Computer neurons dynamics modeling when exposed to constant external signal was conducted in Van der Pol approaching [12]. The authors [13] investigated frequency-temporal dynamics of sensory neuron (threshold device) using the technology of double wavelet analysis and taking into account the interaction of its own dynamics and the dynamics that was caused by the influence of external non-stationary signal. In this sensory neuron was modeled as a threshold device that converts the input signal to pulse sequence output. This pulse sequence was described by the sequence of Dirac delta functions, each of which corresponds to the pulse (adhesions) generation moment. These model pulses have the same shape and amplitude, that's why information about the external effect of dynamic signal appears only in the time intervals between the moments of their generation.

\section{SetTing ObJectives}

The aim of this work is to study the analytical and numerical method of time-frequency and timedependence of the output signal morphology for nonlinear oscillator neurons based on Van der Pol model taking to account threshold effect neurons when it is exposed to different in shape, frequency and amplitude of external non-stationary signals.

\section{MATHEMATICAL MODEL}

Nonlinear oscillator sensory neuron type Van der Pol (threshold device) with its own dynamics $\omega_{0 k}$, which can generate pulses in the absence of external non-stationary signals $\left(V_{k}(t)=0\right)$, when the number of available sensory neuron impulses $N_{0 k}$ reaches a threshold value $N_{c k}$ $\left(N_{0 k}>N_{c k}\right)$ was considered. Thus, such neuron can be considered as a threshold device that converts the input non-stationary signal $V_{k}(t)$ into a sequence of pulses output (Fig.1) due to the "imposition" of the dynamics of input non-stationary signal $V_{k}(t)$ on its own neuron dynamic. Consequently, the signals conversion process analysis by nonlinear oscillator sensory neuron is complicated. The complex dynamics of transformation of input non-stationary signal received by biological sensor with its own dynamics shows an experimental record signal (Fig.1b) [13]-[16]. This signal generated by a biological neuron without external signal action (interval $0<\mathrm{t}<110$ s with a low- $\delta$-pulse sequence). At the time interval $110 \mathrm{~s}<\mathrm{t}<200$ s shown on (Fig. 1b) the result of external signal interaction with the biological sensor with its own dynamics which leads the formation of high-order $\delta$-pulses sequence.

To illustrate, let us consider encoding information by touch nonlinear oscillator k-neuron which describes the nonlinear equation of the form

$$
\ddot{X}_{k}+\mu_{k}\left[X_{k}^{2}-p_{k}^{2}\left(N_{0 k} ; N_{c k}\right)\right] \dot{X}_{k}+\omega_{0 k}^{2} X_{k}=V_{k}(t)
$$

where $p_{k}^{2}\left(N_{0 k} ; N_{c k}\right)=p_{0 k}^{2} \tanh \left(\frac{N_{0 k}-N_{c k}}{\sigma_{k}^{2}}\right)-$ setting the amplitude of the $k$-th neuron; $\mu_{k}>0$;

$N_{0 k}, N_{c k}, \sigma_{k}^{2}$ are the number of pulses that come to $k$-th neuron, threshold pulse $k$-th neuron and variance respectively;

$\omega_{0 k}^{2}$ is natural frequency $k$-th nonlinear oscillator neuron;

$V_{k}(t)$ is non-stationary input signal goes to $k$-th neuron.

Nonlinear oscillator neuron has its own dynamics and generates pulses in the absence of external signals at $N_{0 k}>N_{c k}$, because under this condition $\tanh \left(\frac{N_{0 k}-N_{c k}}{\sigma_{k}^{2}}\right)>0$ and accordingly $p_{k}^{2}\left(N_{0 k} ; N_{c k}\right)>0$ [14]. Mathematical model (1) can also be used to study the collective behavior of ensembles of neurons interconnected synaptic connections $\lambda_{j k}$. For this purpose the second and third terms are necessary to replace $X_{k} \rightarrow X_{\alpha k}$ where $X_{\alpha k}=X_{k}+\sum_{j=1}^{N} \lambda_{j k} X_{j} ; \alpha=1,2, \ldots, \mathrm{N}$.

Solution to equation (1) with the analytical numerical method and consistent approximation method in the form

$$
X_{k}^{(n)}(t)=a_{k}^{(n)}(t) \sin \psi_{k}^{(n)}(t)
$$

where $n=1,2,3, \ldots, N$ is an iteration number;

$$
\psi_{k}^{(n)}(t)=\omega_{0 k} t+\varphi_{k}^{(n)}(t)
$$




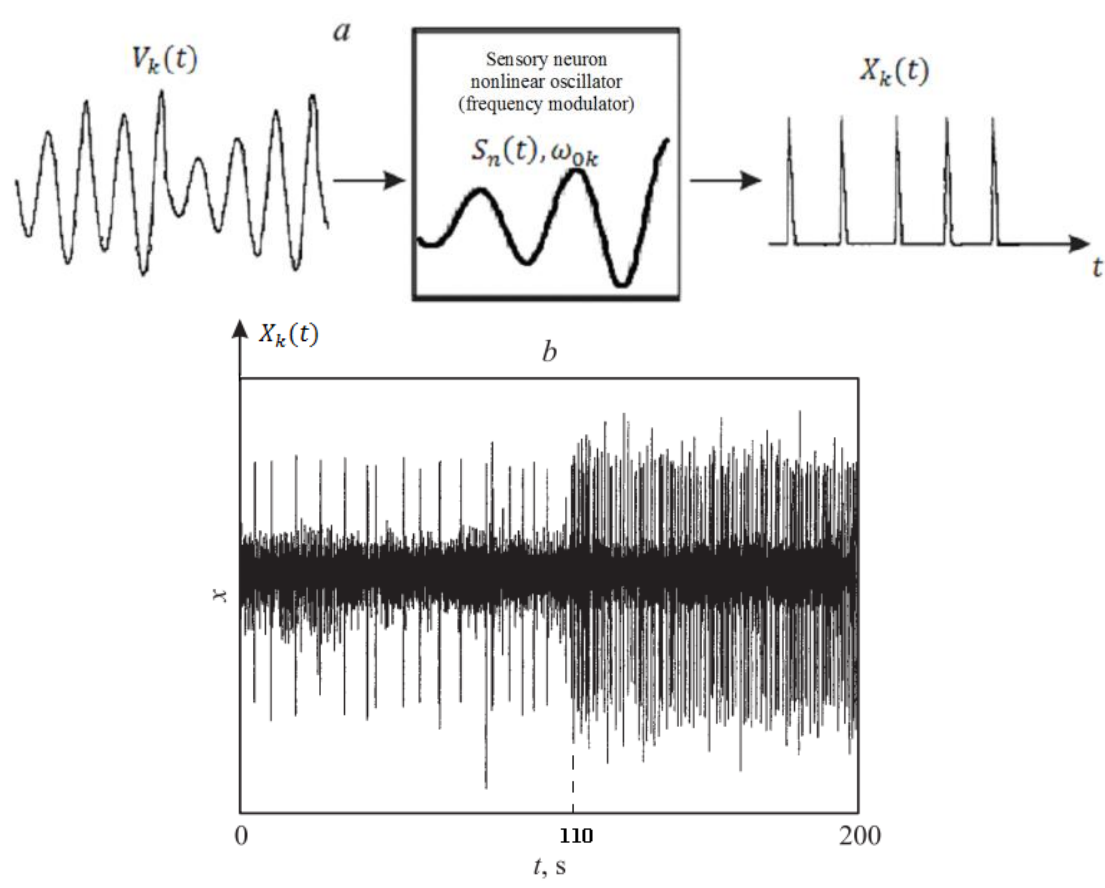

Fig.1. a - Schematic representation of process for the input signal $V_{k}(t)$ conversion by non-linear oscillator sensory neuron (threshold device). Pulses generating times on the output of threshold device $X_{k}(t)$ correspond to the crossing moments of the threshold level; b - An experimental sample recording of signal which is generated by biological neurons [13].

$a_{k}^{(n)}(t)$ and $\varphi_{k}^{(n)}(t)$ - functions of time, which are selected so that the ratio (2) satisfies the equation (1). In addition we impose the condition that $a_{k}^{(n)}(t)$ is a slowly variable function, i.e.

$$
\lim _{t \rightarrow \infty} \frac{a_{k}^{(n)}\left(p_{k} \mu_{k} t\right)}{a_{k}^{(n)}\left(p_{k} t\right)}=1
$$

But since there are two functions $a_{k}^{(n)}(t)$ and $\psi_{k}^{(n)}(t)$, and one equation, this condition ambiguous defines the function. We demand that the condition was fulfilled

$$
\dot{X}_{k}^{(n)}=\omega_{0 k} a_{k}^{(n)}(t) \cos \psi_{k}^{(n)}(t)
$$

where $\dot{X}_{k}^{(n)}=\frac{d X_{k}^{(n)}(t)}{d t}$.

Substituting (2) in (1) and given the condition (5) we obtain the system of equations $a_{k}^{(n)}(t)$ and $\psi_{k}^{(n)}(t)$ :

$$
\left\{\begin{array}{c}
\frac{d a_{k}^{(n)}(t)}{d t}=-a_{k}^{(n-1)}(t) \mu_{k}\left[\left(a_{k}^{(n-1)}\right)^{2} \sin ^{2} \psi_{k}^{(n-1)}(t)-p_{k}^{2}\left(N_{0 k} ; N_{c k}\right)\right] \cos ^{2} \psi_{k}^{(n-1)}(t)+V_{k}(t) \cos \psi_{k}^{(n-1)}(t) \\
\frac{d \psi_{k}^{(n)}(t)}{d t}=\omega_{0 k}+\mu_{k}\left[\left(a_{k}^{(n-1)}\right)^{2} \sin ^{2} \psi_{k}^{(n-1)}(t)-p_{k}^{2}\left(N_{0 k} ; N_{c k}\right)\right] \sin \psi_{k}^{(n-1)}(t) \cos \psi_{k}^{(n-1)}(t)-\frac{V_{k}(t) \sin \psi_{k}^{(n-1)}(t)}{a_{k}^{(n-1)}(t)}
\end{array}\right.
$$

The right side of the equation system (6) for the period $2 \pi$ at $V_{k}(t)=0$ for the rule [15] was averaged:

$$
<\Phi>=\frac{1}{2 \pi} \int_{0}^{2 \pi} \Phi\left(\psi_{k}^{(n)}\right) d \psi_{k}^{(n)}
$$

In zero approximation expressions for $a_{k}^{(0)}(t)$ and $\psi_{k}^{(0)}(t)$ are found from the system of equations:

$$
\frac{d a_{k}^{(0)}(t)}{d t}=A\left(a_{k}^{(0)}(t)\right), \frac{d \psi_{k}^{(0)}(t)}{d t}=B\left(a_{k}^{(0)}(t)\right)
$$

where $A\left(a_{k}^{(0)}(t)\right)=-a_{k}^{(0)}(t) \mu_{k}\left(\frac{\left(a_{k}^{(0)}\right)^{2}}{8}-\frac{p_{k}^{2}}{2}\right), B\left(a_{k}^{(0)}(t)\right)=\omega_{0 k}$.

Integrating the equation (8), a zero approximation expressions for $a_{k}^{(0)}(t)$ and $\psi_{k}^{(0)}(t)$ were worked out:

$$
\begin{gathered}
a_{k}^{(0)}(t)=\frac{2 p_{k}}{\sqrt{1+e^{-p_{k}^{2} \mu_{k} t}}}, \\
\psi_{k}^{(0)}(t)=\omega_{0 k} t
\end{gathered}
$$


where $a_{k}^{(0)}(t)$ satisfies the criterion (4) of slowly variable function. To find expressions $a_{k}^{(1)}(t)$ and $\psi_{k}^{(1)}(t)$ in the first approximation $(n=1)$ we should substitute the expression (9), (10) into the system of equations (6). As a result of integrating we obtain first approximation expressions for $a_{k}^{(1)}(t)$ and $\psi_{k}^{(1)}(t)$. The process of iteration stops when the conditions are fulfilled:

$$
\left|\frac{a_{k}^{(\mathrm{n})}(t)-a_{k}^{(\mathrm{n}-1)}(t)}{a_{k}^{(\mathrm{n})}(t)}\right|<<1,\left|\frac{\psi_{k}^{(\mathrm{n})}(t)-\psi_{k}^{(\mathrm{n}-1)}(t)}{\psi_{k}^{(\mathrm{n})}(t)}\right|<<1 .
$$

\section{RESULTS}

For modeling example, in this problem external nonstationary signal $V_{k}(t)$ is chosen as a sum of $N$ ordinary non-stationary signals, each of which is centered at the point $t=t_{L}$ and characterized by system parameters $L$ [16]

$$
V_{k}(t)=\sum_{L=L_{0}}^{N-1} \lambda_{L k} v_{L k}\left(t-t_{L}\right)+\lambda_{0 k}
$$

By selecting system parameters L, we can to construct mathematical models of complex non-stationary signal $V_{k}(t)$, spectral properties of which change over time. The mathematical model of unsteady external signal $V_{k}(t)$ will reflect the dynamics of the real signal that characterizes a physical (biological) process.

An example of a simple non-stationary signal $v_{L k}\left(t-t_{L}\right)$ is an expression:

$$
v_{L k}\left(t-t_{L}\right)=\frac{1}{2 \tau_{L} \sqrt{\pi}} \exp \left[-\frac{\left(t-t_{L}\right)^{2}}{4 \tau_{L}^{2}}\right] \cos \left(\omega_{L}\left(t-t_{L}\right)+\alpha_{L}\right),
$$

which is a product of the envelope Hauss form to oscillating function and is described by five parameters $L$

$$
L=\left(\lambda_{L k}, \omega_{L}, t_{L}, \tau_{L}, \alpha_{L}\right)
$$

where $\lambda_{L k}$ denotes weight connections of inputs $v_{1}, \ldots, v_{N}$ of the $k$-th neuron; $\lambda_{0 k}$ is weight shift signal of communication with the $k$-th neuron; $\omega_{L}=2 \pi f_{L}$ is external carrier frequency oscillation in hertz $(\mathrm{Hz}), t_{L}$ is center localization signal by the time in seconds, $\tau_{L}$ is typical localization signal time interval in seconds, $\alpha_{L}$ is the initial phase in radians.

Equation (11) with (12) describes all inputs, including offset signal, coming with weights $\lambda_{L k}$ the adder $k$-th neuron (incoming operator $\tilde{f}_{i n}$ ). Input operator $\tilde{f}_{i n}$ converts weighted weights $\lambda_{L k}$ inputs and presents them to the operator activation $\tilde{f}_{a}$ (fig.2). For nonlinear oscillator sensory neuron activation operator $\tilde{f}_{a}$ looks like, this input operator

$$
\tilde{f}_{a}=\frac{d^{2}}{d t^{2}}+\mu_{k}\left[X_{k}^{2}-p_{k}^{2}\left(N_{0 k} ; N_{c k}\right)\right] \frac{d}{d t}+\omega_{0 k}^{2} .
$$

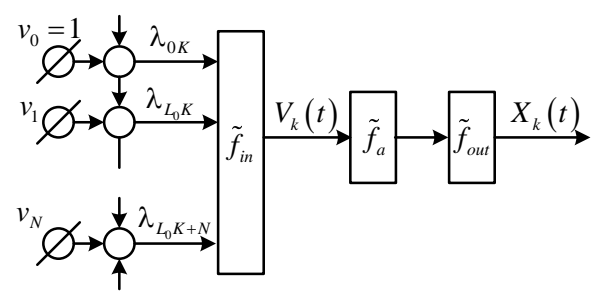

Fig.2. The structure of artificial nonlinear oscillator neuron

The output signal of nonlinear oscillator neuron $X_{k}(t)$ (Fig.2) is transformed by the source operator $\tilde{f}_{\text {out }}$ the output signal of service activation. Output operator $\tilde{f}_{\text {out }}$ is required to represent the state of the neuron in the desired field values. In most studies, this operator is not isolated, and under output signal the neuron to understand the signal after activation operator $\tilde{f}_{a}$. However, during the analysis and synthesis of artificial neural networks (ANN), which have different activation functions of the various regions and areas of value determination, is necessary taking into account the output operator. Consequently, nonlinear operator transformation of input signals $V_{k}(t)$ vector in the vector output signal $X_{k}(t)$ can be written as

$$
X_{k}(t)=\tilde{f}_{\text {out }}\left(\tilde{f}_{a}\left(\tilde{f}_{\text {in }}\left(V_{k}(t), \lambda_{\text {Lk }}\right)\right)\right)
$$

By selecting different combinations of $L=\left(\lambda_{L k}, \omega_{L}, t_{L}, \tau_{L}, \alpha_{L}\right)$, we can construct a theoretical model that adequately describes the real physical (biological) processes in the interaction of external nonstationary signal $V_{k}(t)$ with its own dynamic physical (biological) nonlinear neuron.

\section{DISCUSSION}

Figs. 3-4 shows a first term $(\mathrm{N}=1)$ non-stationary external signal $V_{k}(t)((11),(12))$ depending on the time

$$
v_{L_{0} k}\left(t-t_{L_{0}}\right)=\frac{\lambda_{L_{0} k}}{2 \tau_{L_{0}} \sqrt{\pi}} \exp \left[-\frac{\left(t-t_{L_{0}}\right)^{2}}{4 \tau_{L_{0}}^{2}}\right] \cos \left(\omega_{L_{0}}\left(t-t_{L_{0}}\right)+\alpha_{L_{0}}\right),
$$

applied to nonlinear oscillator sensory neuron for two parameter values $L_{0}: L_{0}=(3,4 \pi, 12,3,0) ; L_{0}=(1$, $6 \pi, 21,1,0)$ in accordance. In the first case the signal $v_{L_{0} k}\left(t-t_{L_{0}}\right)$ (Fig.3) has an amplitude $\lambda_{L_{0} k}=3$, and the 
second $-\lambda_{L_{0} k}=1$ respectively, in the first case, the signal has a frequency $f_{L_{0}}=2 \mathrm{~Hz}$, centered at a point in time $t_{L_{0}}=12 \mathrm{~s}$ with time-lapse localization signal $\tau_{L_{0}}=3 \mathrm{~s}$ and

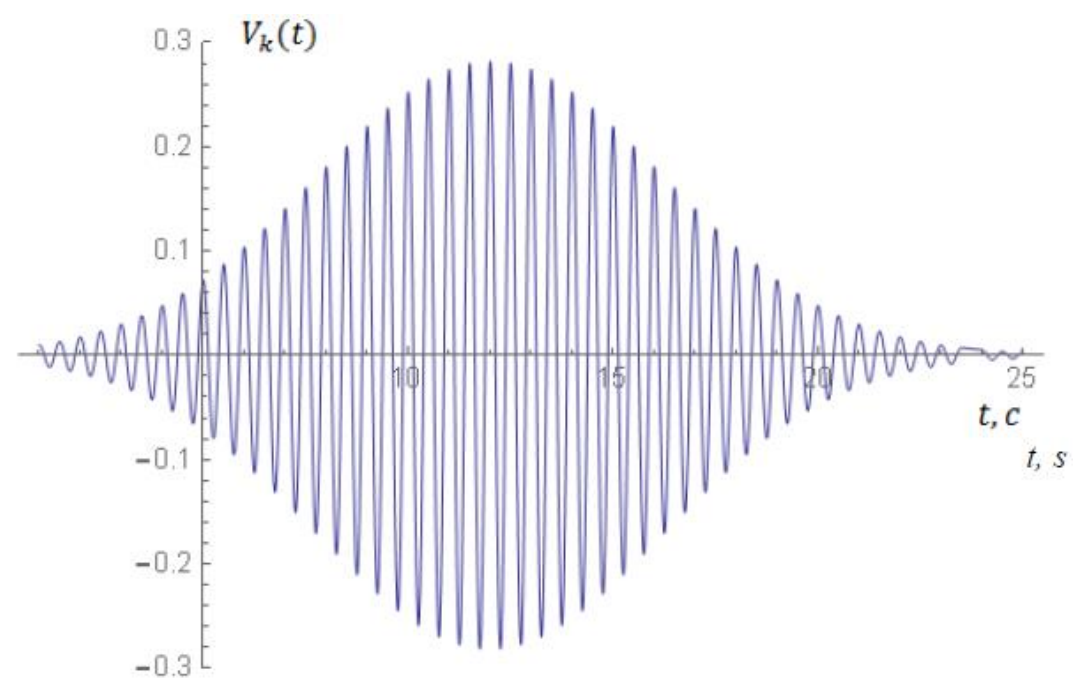

Fig.3. Morphology of the external information signal $v_{L_{0} k}\left(t-t_{L_{0}}\right)$ (16) with parameter values $L_{0}=(3,4 \pi, 12,3,0) ; p_{k}=0.4$ and $\mu_{k}=0.1$.

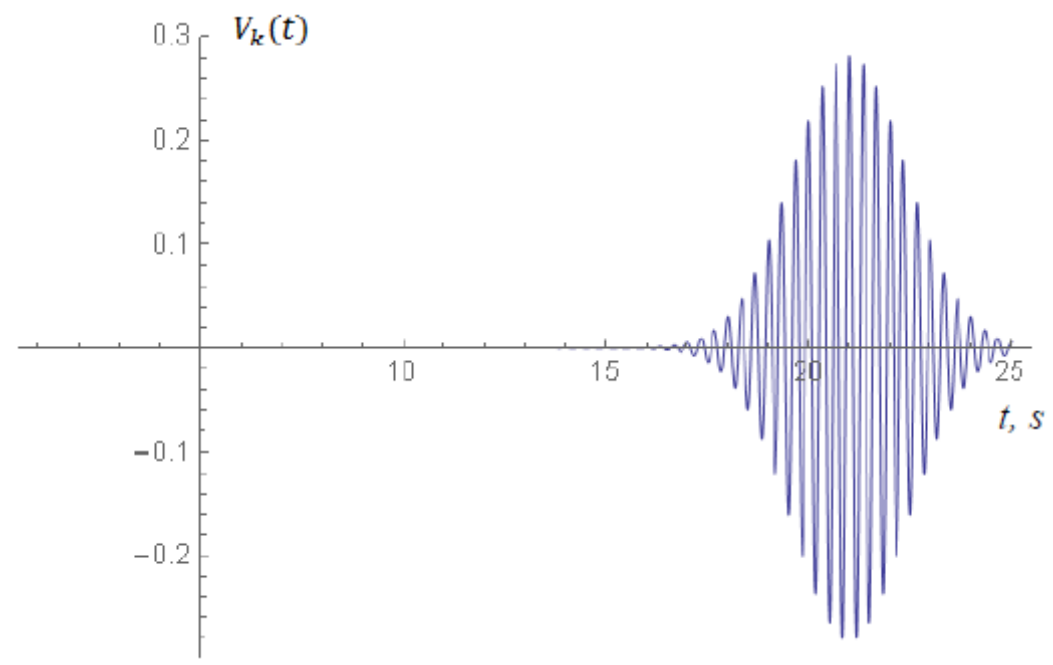

Fig.4. Morphology of the external information signal $v_{L_{0} k}\left(t-t_{L_{0}}\right)$ (16) with parameter values $L_{0}=(1,6 \pi, 21,1,0) ; p_{k}=0.4$ and $\mu_{k}=0.1$.

In numerical calculations, signals $X_{k}(t)$ the output of the nonlinear oscillator neuron with input signals (Figs. 34) parameters $p_{k}=0.4$ and $\mu_{k}=0.1$ match.

Figs. 5-6 present graphs of frequency modulation $\omega_{k}^{(n)}=\frac{d \psi_{k}^{(n)}(t)}{d t}$, which changes the instantaneous frequency of the carrier oscillation information $V_{k}(t)$ the initial phase $\alpha_{L_{0}}=0$, and the second $-f_{L_{0}}=3 \mathrm{~Hz}$; $t_{L_{0}}=21 \mathrm{~s}, \tau_{L_{0}}=3 \mathrm{~s} ; \alpha_{L_{0}}=0$. 


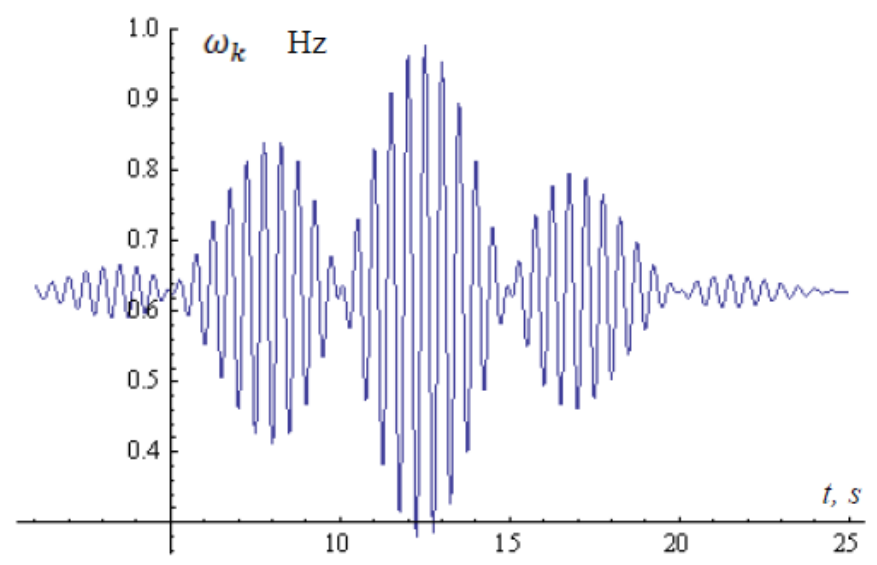

Fig.5. The time dependence of the instantaneous frequency of the carrier signal information $v_{L_{0} k}\left(t-t_{L_{0}}\right)(16)$ with parameter values $L_{0}=(3,4 \pi, 12,3,0) ; p_{k}=0.4$ and $\mu_{k}=0.1, \omega_{0 k}=0.2 \pi$.

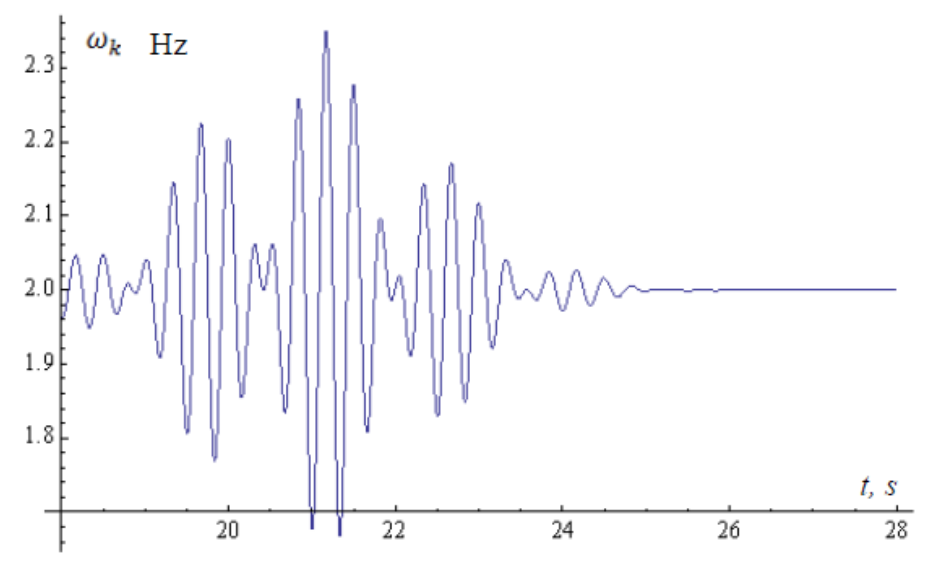

Fig.6. The time dependence of the instantaneous frequency of the carrier signal information $v_{L_{0} k}\left(t-t_{L_{0}}\right)(16)$ with parameter values $L_{0}=(1,6 \pi, 21,1,0) ; p_{k}=0.4$ and $\mu_{k}=0.1, \omega_{0 k}=2$

Figs. 7-8 present graphs of morphology signal at the output of the nonlinear oscillator neuron $X_{k}(t)$ defined by the nature of the interaction of dynamics neuron with frequencies $\omega_{0 k}=0.2 \pi ; \omega_{0 k}=2$ and dynamic, caused by external influence $v_{L_{0} k}\left(t-t_{L_{0}}\right)(16)$.

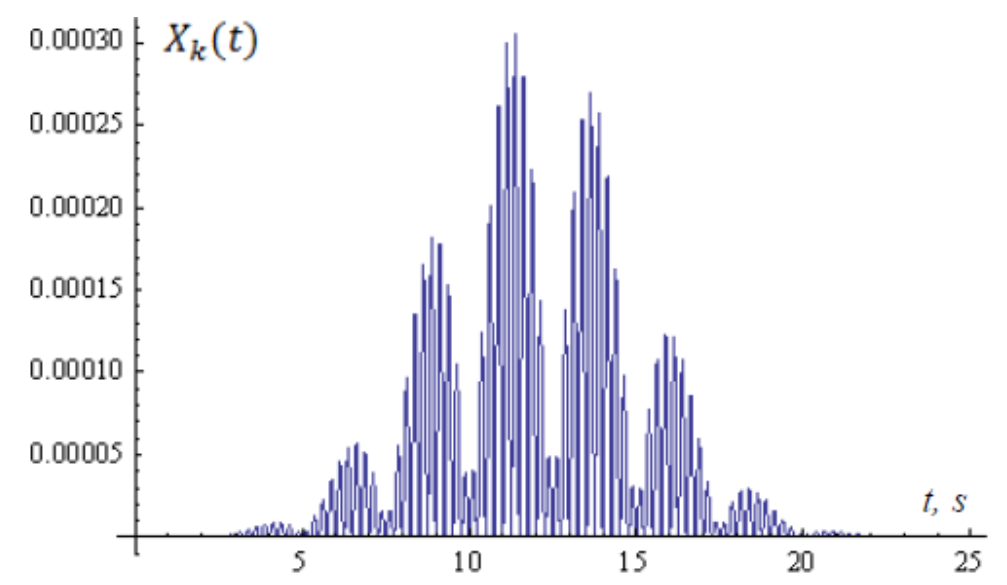

Fig.7. Schedule of morphology signal at the output of the nonlinear oscillator neuron $X_{k}(t)$ with parameter values $L_{0}=(3,4 \pi, 12,3,0) ; p_{k}=0.4$ and $\mu_{k}=0.1, \omega_{0 k}=0.2 \pi$. 


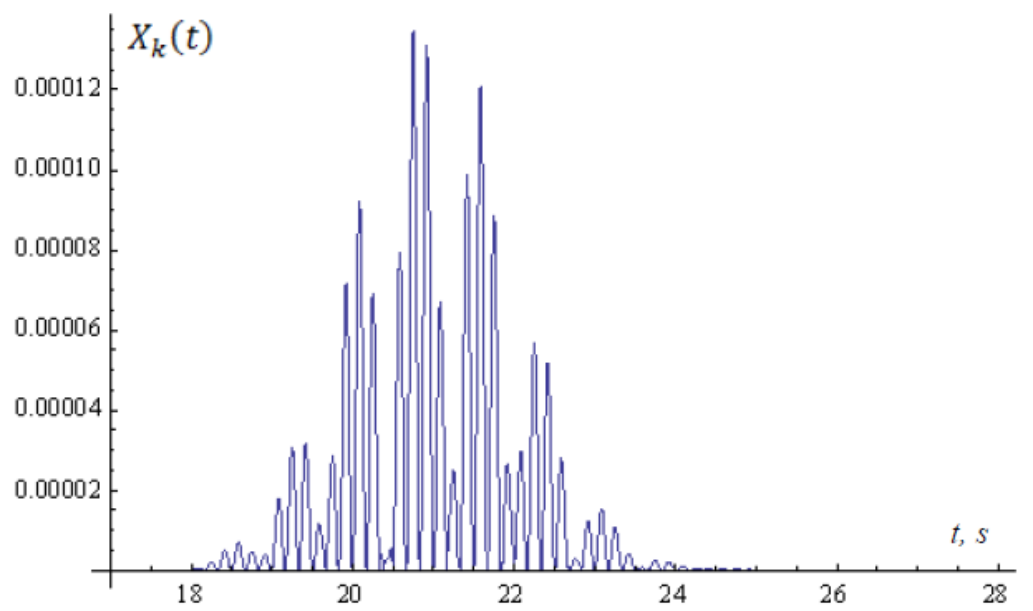

Fig.8. Schedule of morphology signal at the output of the nonlinear oscillator neuron $X_{k}(t)$ with parameter values $L_{0}=(1,6 \pi, 21,1,0) ; p_{k}=0.4$ and $\mu_{k}=0.1, \omega_{0 k}=2$.

Figs. 9-10 present graphs of morphology signal at the output of the nonlinear oscillator neuron $X_{k}(t)$, defined by the nature of the interaction of dynamics neuron with frequencies $\omega_{0 k}=0.2 \pi ; \omega_{0 k}=2$ and dynamic, caused by external influence $v_{L_{0} k}\left(t-t_{L_{0}}\right)(16)$.

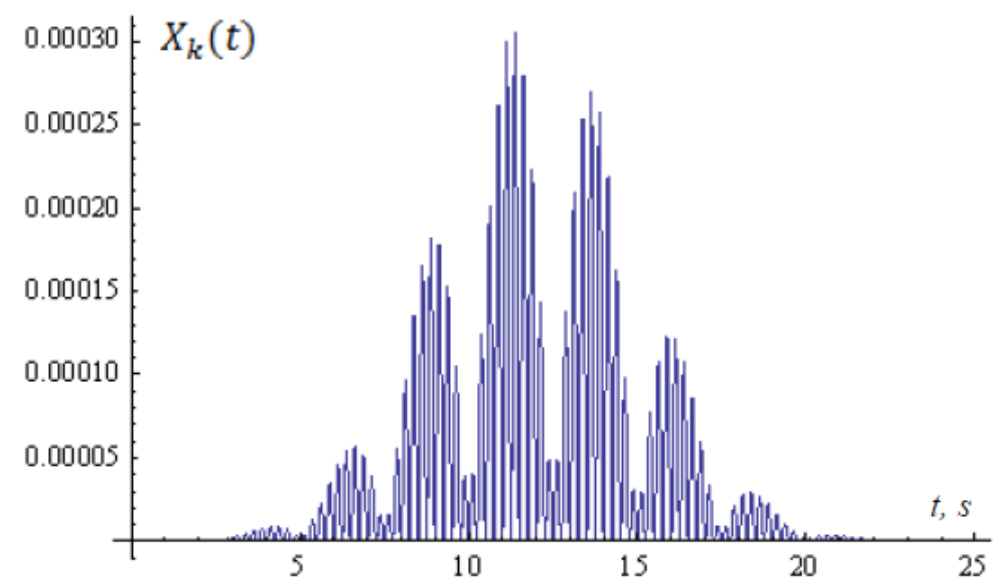

Fig.9. Schedule of morphology signal at the output of the nonlinear oscillator neuron $X_{k}(t)$ with parameter values $L_{0}=(3,4 \pi, 12,3,0) ; p_{k}=0.4$ and $\mu_{k}=0.1, \omega_{0 k}=0.2 \pi$.

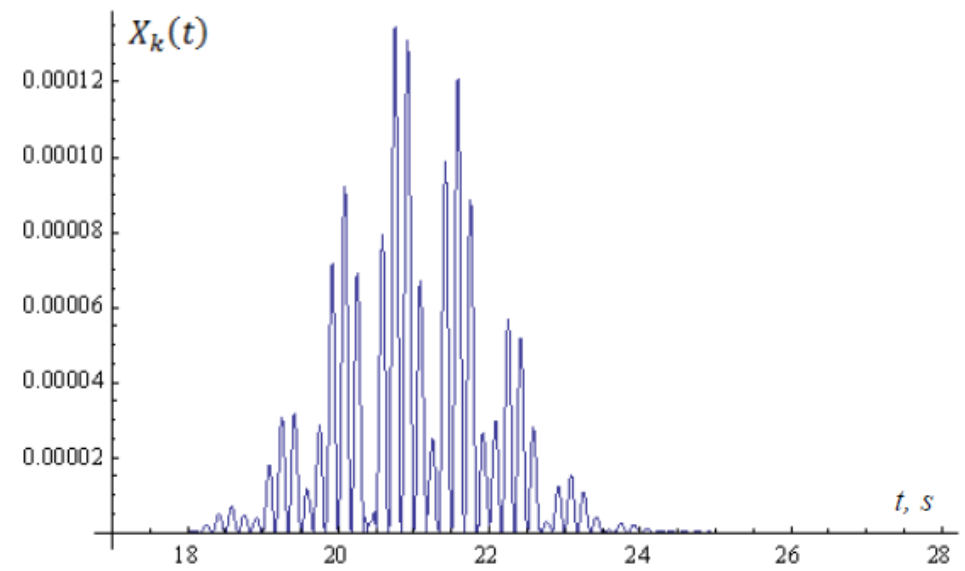

Fig.10. Schedule of morphology signal at the output of the nonlinear oscillator neuron $X_{k}(t)$ with parameter values $L_{0}=(1,6 \pi, 21,1,0) ; p_{k}=0.4$ and $\mu_{k}=0.1, \omega_{0 k}=2$. 
The interaction of external signal $v_{L_{0} k}\left(t-t_{L_{0}}\right)$ with parameters $L_{0}=(3,4 \pi, 12,3,0) ; L_{0}=(1,6 \pi, 21,1,0)$ with its own dynamics of nonlinear oscillator neuron $\omega_{0 k}=4 \pi$ or $\omega_{0 k}=6 \pi$ a sharp increase in the amplitude of the output signal $X_{k}(t)$ (Fig. 11) compared to the output signal in Fig.9. That is a resonance effect in the external signal frequency which matches the natural frequency of oscillation of the nonlinear neuron, $\left(\omega_{L_{0}}=\omega_{0 k}\right)$. Graph of modulation frequency $\omega_{k}^{(n)}(t)$ if a resonance effect is shown in Fig.12.

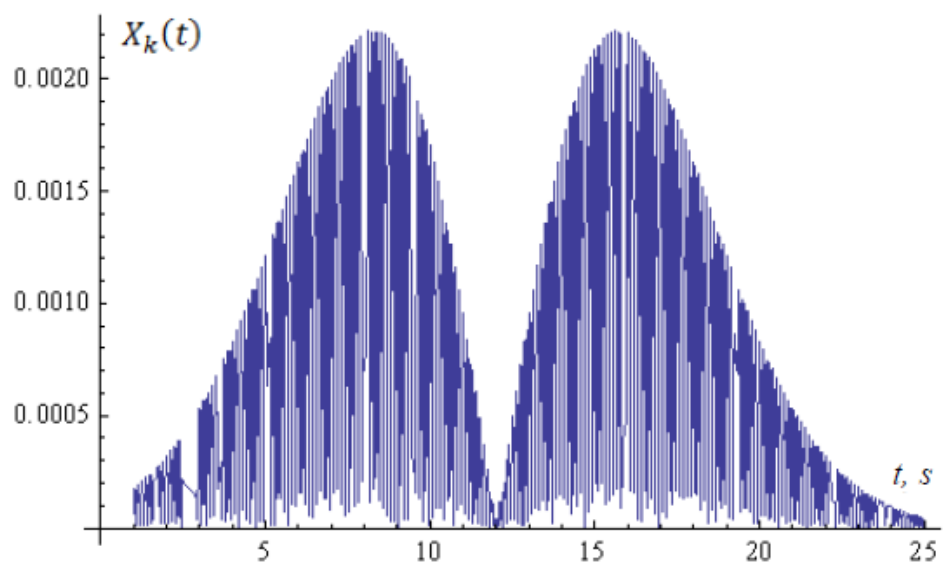

Fig.11. Schedule of morphology signal at the output of the nonlinear oscillator neuron $X_{k}(t)$ if a resonance effect with parameter values $L_{0}=(3,4 \pi, 12,3,0) ; p_{k}=0.4$ and $\mu_{k}=0.1, \omega_{0 k}=4 \pi$.

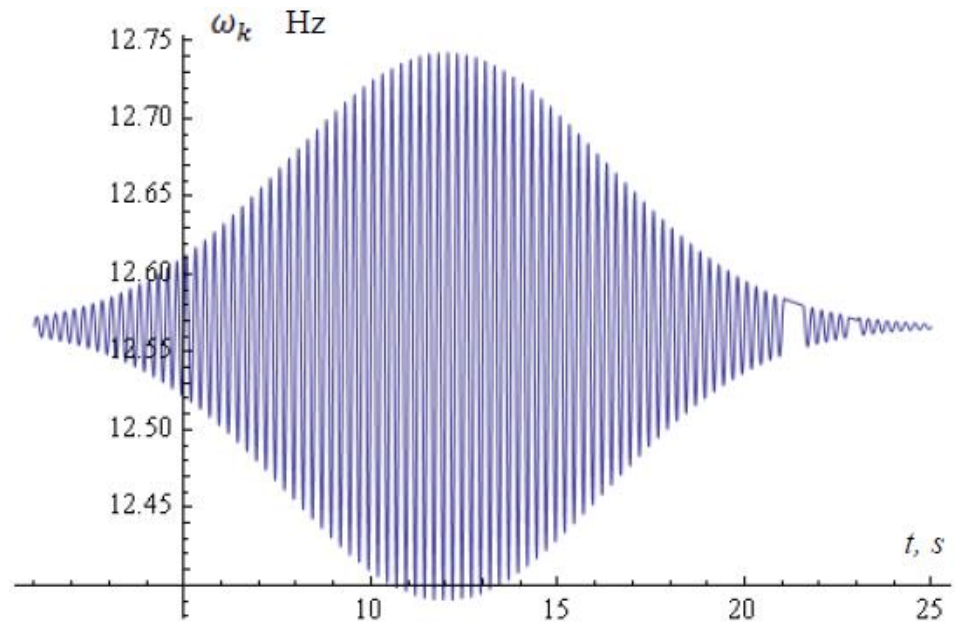

Fig.12. The time dependence of the instantaneous frequency of the carrier signal information $v_{L_{0} k}\left(t-t_{L_{0}}\right)$ (16) if a resonance effect with parameter values $L_{0}=(3,4 \pi, 12,3,0) ; p_{k}=0.4$ and $\mu_{k}=0.1, \omega_{0 k}=4 \pi$.

To decode the structure of output signal $X_{k}(t)$, it is important to know the code of program, which describes the inverse operator acting on a vector output, i.e.

$$
\tilde{f}_{\text {in }}^{-1}\left(\tilde{f}_{a}^{-1}\left(\tilde{f}_{\text {out }}^{-1} X_{k}(t)\right)\right)=V_{k}\left(t, \lambda_{L k}\right)
$$

Thus, the received results allow hypothesizing that the process of encoding information of nonlinear neurons can be considered in terms of modulation frequency as we know in radio physics that modulation frequency is one way of information transmitting.

\section{CONCLUSIONS}

The method of inhomogeneous nonlinear differential equations with quadratic non linearity of the unknown function at the first derivative was suggested.

It was defined that nonlinear oscillator neuron can act as a frequency modulator, which can modulate the input information of non-stationary signal.

It was determined that nonlinear oscillator neuron with threshold effect significantly alters the structure of the input information non-stationary signal different in shape, frequency and amplitude. 
The existence of resonance effects in nonlinear oscillator neuron when frequency of the external nonstationary signal and dynamics of the natural frequency of the neuron are equal is determined.

Information coding with nonlinear oscillator neuron on the basis of frequency modulation and decoding using an inverse operator, which acts on the output signal vector were suggested.

\section{REFERENCES}

[1] T. Sauer, "Reconstruction of dynamical systems from interspike intervals." Phys. Rev. Lett., Vol. 72, pp. 38113814, 1994

[2] D.M. Racicot, A. Lonytin, "Interspike interval attractors from chaotically driven neuron models." Physic D., Vol. 104, pp. 184-204, 1997.

[3] R. Castro, T. Sauer, "Correlation dimension of attractors through inters pike intervals." Phys. Rev. E., Vol. 55, pp. 287-290, 1997.

[4] T. Sauer, "Nonlinear Dinamics and Time Series." Eds C. Culter and D. Kaplan, Fields Institute Communications. American Math. Society, Providence, RI, Vol. 11, pp. 6375, 1997.

[5] R. Hegger, H. Kantz, "Embedding of sequences of time intervals." Europhys. Lett., Vol. 38, pp. 267-272, 1997.

[6] R. Castro, T. Sauer, "Chaotic Stochastic Resonance: Noise-Enhanced Reconstruction of Attractors." Phys. Rev. Lett., Vol. 79, pp. 1030-1033, 1997.

[7] A. Pavlov, A. Khramov, A. Koronovskyy, E. Sytnykova, V. Makarov, A. Ovchynnykov, "Veyvlet-analyz v neyrodynamyke." Uspekhy fyzycheskykh nauk, Vol. 182(9), pp. 905-939, 2012.

[8] H.C. Tuckwell, "Introduction to Theoretical Neurobiology." Cambridge University. Press, Cambridge, 1998.

[9] N. Janson, A. Pavlov, A. Neiman, V. Anishchenko, "Reconstruction of dynamical and geometrical properties of chaotic attractors from threshold-crossing interspike intervals." Phys. Rev. E., Vol. 58, pp. R4-R7, 1998.

[10] A. Pavlov, O. Sosnovtseva, E. Mosekilde, V. Anishchenko, "Extracting dynamics from thresholdcrossing interspike intervals: Possibilities and limitations." Phys. Rev. E., Vol. 61, pp. 5033-5044, 2000.

[11] A. Pavlov, O. Sosnovtseva, E. Mosekilde, V. Anishchenko, "Chaotic dynamics from interspike intervals." Phys. Rev. E., Vol. 63, pp. 036205(5), 2001.

[12] S. John, Hooshang Hemami Bay, "Modeling of a Neural Generator with Coupled Nonlinear Oscillators." IEE Transactions biomedical engineering, V.BME, Vol. 34(4), pp. 297-306, 1987.

[13] A. Pavlov, O. Pavlova, "Prymenenye veyvlet-analyza v yssledovanyyakh struktury tochechnykh protsessov." Pys'ma v ZHTF, Vol. 32(21), pp. 11-17, 2006.

[14] V.Y. Suhakov, “Osnovy synerhetyky.”, Kyyiv, 2001.

[15] M. Boholyubov, Y. Mytropolskyy, "Asymptotychni metody v teoriyi neliniynykh rivnyan", M.: Nauka, 1992.

[16] S.V. Bozhokyn, "Nepreryvnoe veyvlet-preobrazovanye y tochno reshaemaya model' nestatsyonarnykh syhnalov." ZHTF, Vol. 82(7), pp. 8-13. 2012.

[17] T. Basyuk, "The main reasons of attendance falling of internet resource.", in Proc. of the X-th Int. Conf. Computer Science and Information Technologies, CSIT'2015, pp. 91-93, 2015.

[18] E. Burov, "Complex ontology management using task models." International Journal of Knowledge-Based and
Intelligent Engineering Systems, Amsterdam: IOS Press, Vol. 18(2), pp. 111-120, 2014.

[19] V. Lytvyn, V. Vysotska, L. Chyrun, L. Chyrun, "Distance Learning Method for Modern Youth Promotion and Involvement in Independent Scientific Researches." in Proc. of the First International Conference on Data Stream Mining \& Processing, DSMP, pp. 269-274, 2016.

[20] J. Chen, D. Dosyn, V. Lytvyn, A. Sachenko, "Smart Data Integration by Goal Driven Ontology Learning." Advances in Big Data. Advances in Intelligent Systems and Computing, Springer, pp. 283-292, 2016.

[21] V. Lytvyn, V. Vysotska, O. Veres, I. Rishnyak, H. Rishnyak, "Classification Methods of Text Documents Using Ontology Based Approach." Advances in Intelligent Systems and Computing, Vol. 512, Springer, pp. 229-240, 2016.

[22] V. Teslyuk, V. Beregovskyi, P. Denysyuk, T. Teslyuk, A. Lozynskyi, "Development and Implementation of the Technical Accident Prevention Subsystem for the Smart Home System." International Journal of Intelligent Systems and Applications (IJISA), Vol. 10, No. 1, pp. 1-8, 2018.

[23] Zh. Hu, Ye.V. Bodyanskiy, O.K. Tyshchenko, and V.O. Samitova,"Fuzzy Clustering Data Given in the Ordinal Scale", International Journal of Intelligent Systems and Applications (IJISA), Vol.9, No.1, pp.67-74, 2017.

[24] Zh. Hu, Ye.V. Bodyanskiy, O.K. Tyshchenko, V.O. Samitova,"Possibilistic Fuzzy Clustering for Categorical Data Arrays Based on Frequency Prototypes and Dissimilarity Measures", International Journal of Intelligent Systems and Applications (IJISA), Vol.9, No.5, pp.55-61, 2017.

[25] Zh. Hu, Ye.V. Bodyanskiy, O.K. Tyshchenko, V.M. Tkachov, "Fuzzy Clustering Data Arrays with Omitted Observations", International Journal of Intelligent Systems and Applications (IJISA), Vol.9, No.6, pp.24-32, 2017.

[26] Zh. Hu, Ye.V. Bodyanskiy, O.K. Tyshchenko, and V.O. Samitova,"Fuzzy Clustering Data Given on the Ordinal Scale Based on Membership and Likelihood Functions Sharing", International Journal of Intelligent Systems and Applications (IJISA), Vol.9, No.2, pp.1-9, 2017.

[27] J. Su, V. Vysotska, A. Sachenko, V. Lytvyn, Y. Burov, "Information resources processing using linguistic analysis of textual content." in Proc. of the 9th IEEE International Conference Intelligent Data Acquisition and Advanced Computing Systems: Technology and Applications (IDAACS), Bucharest, Romania, pp. 573578, 2017.

[28] V. Lytvyn, V. Vysotska, Y. Burov, O. Veres, I. Rishnyak, "The Contextual Search Method Based on Domain Thesaurus." Advances in Intelligent Systems and Computing, Vol. 689, Springer, pp. 310-319, 2017.

[29] V. Pasichnyk, T.Shestakevych, "The model of data analysis of the psychophysiological survey results." Advances in Intelligent Systems and Computing, Vol. 512, pp. 271-281, 2017.

[30] P. Zhezhnych, O.Markiv, "Linguistic Comparison Quality Evaluation of Web-Site Content with Tourism Documentation Objects" Advances in Intelligent Systems and Computing, Vol. 689, pp. 656-667, 2018.

[31] O. Bazylyk, P. Taradaha, O. Nadobko, L. Chyrun, T. Shestakevych, "The results of software complex OPTAN use for modeling and optimization of standard engineering processes of printed circuit boards manufacturing" in Proceedings of the 11th International Conference TCSET'2012, pp. 107 - 108, 2012. 
[32] A. Bondariev, M. Kiselychnyk, O. Nadobko, L. Nedostup, L. Chyrun, T. Shestakevych, "The software complex development for modeling and optimizing of processes of radio-engineering equipment quality providing at the stage of manufacture" in Proceedings of the 11th International Conference TCSET'2012, pp. 159, 2012.

[33] V. Riznyk, "Multi-modular Optimum Coding Systems Based on Remarkable Geometric Properties of Space" Advances in Intelligent Systems and Computing, Vol. 512, pp. 129-148, 2017.

\section{Authors' Profiles}

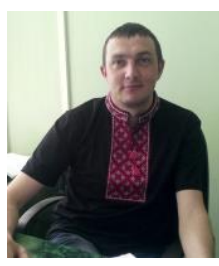

Vasyl Lytvyn was born on January 17, 1976. In 1997 he received the MS from Ivan Franko National University of Lviv, major: applied mathematics. He received the $\mathrm{PhD}$ in Engineering Science and Doctor of Science degrees from Lviv Polytechnic National University, in 2003 and 2012, respectively. He works at Lviv Polytechnic National University as a head of information systems and networks department.

The area of expertise includes construction of subject domain ontologies, methods of knowledge extraction, ontological engineering, and methods of computer-assisted ontology filling, ontology languages, testing of software systems, design process and software management, system analysis.

V.Lytvyn is an author of over 150 scientific papers.

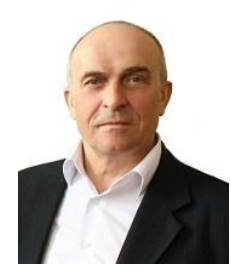

Peleshchak Roman. In 1980 he received the MS from Ivan Franko National University of Lviv, major: radiophysics and electronics. $\mathrm{He}$ received the Doctor of Physical and Mathematical Sciences degrees in 2001. He received the Professor rank in 2002. He works at Ivan Franko Drohobych State Pedagogical University as a head of the Department of General physics. The area of expertise includes construction of theoretical physics, physics of intense nanoheterosystems with different dimensions, nonlinear neural networks, encoding methods that using nonlinear neural networks, pattern recognition, quantum dots in photodynamic therapy. R. Peleshchak is an author of over 320 scientific papers.

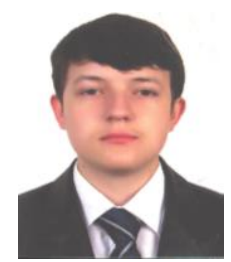

Peleshchak Ivan was born on July 21, 1995. He studies at Lviv Polytechnic National University as a undergraduate student of information systems and networks.

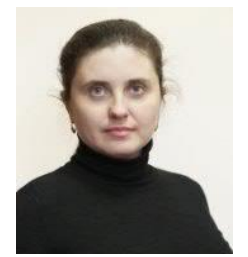

Victoria Vysotska, PhD, Deputy Head of Information systems and networks Department, Associate Professor of Information Systems and Networks Department, Institute of Computer Science and Information Technology at Lviv Polytechnic National University, Lviv, Ukraine. In 2014 defended candidate thesis (PhD) "Methods and tools of information resources processing in the electronic

content commerce systems". Research interests: content, information systems and networks, ecommerce, businessprocess, information resources, commercial content, content analysis, content monitoring, content search, electronic content commerce systems, content management system, content lifecycle, Internet newspaper, software systems, models, algorithms, analysis, methods and strategies of systems design. Victoria has over 18 years of teaching in Lviv Polytechnic National University. She has published more than 270 scientific papers in various national and international journals and conferences, 4 monograph, 5 textbooks. Lector is mathematical linguistics, discrete mathematics and numerical methods in informatics, information resources processing. Information about citations is available in http://orcid.org/0000-0001-64173689, http://victana.lviv.ua/index.php/naukovi-statti or https://scholar.google.com.ua/citations?hl=uk\&user=-

MCARowAAAAJ\&view_op=list_works. Detailed information about Victoria Vysotska can be found on: https://ua.linkedin.com/pub/victoria-vysotska/29/1b7/261.

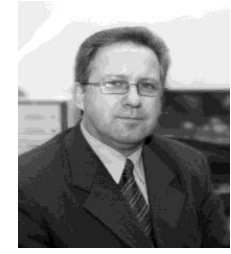

Support Systems
Rishnyak Ihor, Senior lecturer of Information Systems Institute Department in Computer Science and Information Technology at Lviv Polytechnic National University

Research interests: Project management, Project management, Intelligent Decision

How to cite this paper: Vasyl Lytvyn, Victoria Vysotska, Ivan Peleshchak, Ihor Rishnyak, Roman Peleshchak, "Time Dependence of the Output Signal Morphology for Nonlinear Oscillator Neuron Based on Van der Pol Model", International Journal of Intelligent Systems and Applications(IJISA), Vol.10, No.4, pp.8-17, 2018. DOI: 10.5815/ijisa.2018.04.02 